\title{
QUARTERLY OF APPLIED MATHEMATICS
}

\section{RELATIONS BETWEEN THE TRANSMITTING AND RECEIVING PROPERTIES OF ANTENNAS*}

\author{
BY \\ A. F. STEVENSON \\ University of Toronto
}

1. Introduction. It has been known for some time that there exist relations between the transmitting and receiving properties of antennas. Such a relation was first established by Carson, ${ }^{1}$ and results of like character have been given by Ballantine ${ }^{2}$ and Sommerfeld. ${ }^{3}$ Mention may also be made of discussions by Slater ${ }^{4}$ and Schelkunoff. ${ }^{5}$

When critically examined, however, both the statement and the proof of the results claimed leave much to be desired from the point of view of preciseness and rigour. Without attempting a detailed criticism of previous discussions of the subject, it appears to the writer that such discussions are all open to at least some of the following objections.

(1) Results proved for circuits-e.g. Thévenin's theorem-are assumed without proof to apply to antennas.

(2) The boundary conditions to be satisfied at the antenna by the field quantities are not precisely stated.

(3) No distinction is made between an antenna acting as receiver, with connected load impedance, and one acting as a parasite.

(4) The "impressed voltage" is introduced in an unsatisfactory manner which is not consistent with Maxwell's equations. In particular, no account is taken of the "gap."

(5) "Reciprocal theorems" proved for two antennas are assumed to establish results relating to the transmitting and receiving properties for a single antenna. Clearly, it should be possible to establish such results without referring to a second antenna at all.

(6) The antenna is treated as an infinitesimal Hertzian dipole, which is an unnecessary and unwarranted simplification (this applies to Sommerfeld's ${ }^{3}$ work).

In this paper, a discussion of the subject is presented which, it is believed, is more thorough and rigorous than any hitherto given. In Sec. 2 a rigorous formulation, in

* Received Dec. 26, 1946.

1 J. R. Carson, Bell System Tech. J., 3, 393 (1924); 9, 325 (1930); Proc. I.R.E., 17, 952 (1929).

${ }^{2}$ S. Ballantine, Proc. I.R.E., 17, 929 (1929).

${ }^{3}$ A. Sommerfeld, Z. für Hochfrequenztechnik, 26, 93 (1925). See also the article by Sommerfeld in Frank-Mises, Differentialgleichungen der Physik, Rosenberg Publ. N.Y.C., 1943, vol. II, 7th ed., p. 576.

${ }^{4}$ J. C. Slater, Microwave Transmission, McGraw-Hill, 1942, chap. 6.

${ }^{5}$ S. A. Schelkunoff, Electromagnetic Waves, Van Nostrand 1943, p. 476. 
terms of field theory, of three fundamental mathematical problems associated with an antenna, namely those of a transmitting, parasitic, and receiving antenna, is given, it being assumed that the antenna is perfectly conducting. The surrounding medium may be of a very general (linear) type. Attention is confined throughout to a steady state with a definite frequency. In Sec. 3 it is shown that of the three problems mentioned, one, namely that of reception, can be solved very simply in terms of the solutions of the other two. Incidentally, a rigorous proof of Thévenin's theorem for antennas, without any appeal to circuit theory, is given. In Sec. 4 it is shown further that there is a connection between the transmission and parasite problems which, effectively, enables us to solve the latter problem (and hence also the reception problem) once the former has been solved. An application is made to the case where the whole medium is homogeneous and isotropic, and in particular to a linear antenna in such a medium.

In Sec. 5 reciprocal relations between two antennas are discussed. These are of the usual type, though it is believed that they are somewhat more precisely stated than is usually the case. An apparent contradiction of the reciprocal relation which has been put forward by Ballantine ${ }^{2}$ (originally in criticism of Sommerfeld's ${ }^{3}$ work) is here briefly dealt with. In Sec. 6 the question is discussed as to whether the directional properties of an antenna, as exhibited by a polar diagram, are identical for transmission and reception. Adopting a reasonable definition of "polar diagram," it is concluded that the identity of the polar diagrams does not hold in general, although it does so (at least approximately) in cases which are likely to occur in practice. In Sec. 7 the results are extended to cover the case where the antenna is imperfectly conducting. In an appendix a theorem of Lorentz, which is used in the course of the work, is extended to the case where the medium is anisotropic.

The results of Secs. 4 and 5, and subsequent results based on these, involve a certain approximation; but the errors involved would be negligible in practical cases.

2. The three mathematical problems associated with an antenna. We shall consider the antenna to be perfectly conducting, though this restriction will be removed in Sec. 7. The surrounding medium will be supposed to be characterized by linear constitutive relations so that the field equations are all linear. These relations need not, however, unless the contrary is stated, be of the usual isotropic type. We may suppose that the medium is crystalline and is characterized by symmetric dielectric, permeability and conductivity tensors $\epsilon_{i j}, \mu_{i j}, \sigma_{i j}$ whose components are arbitrary functions of position. Further, these tensors may have arbitrary surfaces of discontinuity. ${ }^{6}$ We shall confine ourselves to a steady state solution in which all field quantities vary with the time according to the factor $e^{-i \omega t}$, this factor being omitted, and we shall employ Gaussian units throughout.

We shall, for the sake of generality, consider an "antenna" to be any closed perfectly conducting surface; usually this surface will, of course, take the form of a wire or system of wires. Since the antenna is perfectly conducting, the tangential components of $\mathbf{E}$ vanish everywhere on the antenna surface except where there is an impressed (or induced) E.M.F. or "voltage." Such voltages occur when the antenna is acting as a transmitter or receiver, but not, of course, when it is acting as a parasite. To treat the case where there is a voltage in a reasonably simple manner which en-

${ }^{6}$ There may also be perfect conductors in the surrounding medium $(\sigma=\infty)$. This case can either be treated as a limiting case or considered $a b$ initio. 
ables us to dispense with the connecting circuit (as is always done in such cases), we shall ignore the connecting circuit, but suppose that there is a small "gap" on the antenna surface where the transmitting or receiving circuit is connected. ${ }^{7}$ The component of $\mathbf{E}$ across this gap does not vanish, but rises to a large value, in such a way that the integral of this component taken across the gap defines the voltage. The component of $\mathbf{E}$ "round" the gap, on the other hand, may be supposed to vanish. This "model" is, effectively, that which has been adopted by Stratton and Chu for a perfectly conducting (spherical or spheroidal) antenna, ${ }^{8}$ a detailed discussion of which has recently been given by Infeld. ${ }^{9}$

With an ordinary antenna in the form of a wire, there will be no difficulty in picturing this gap. To include the case where the antenna is of any shape, however, we shall define the gap more precisely as a portion of the antenna surface bounded by two closed curves which are close together. We take two orthogonal families of curves in the gap, the two curves bounding the gap being members of one family. At any point of the gap two directions are thus defined, which we may refer to as "across the gap" and "round the gap." Let these two directions be denoted by $s, s^{\prime}$ respectively, related in such a way that a right-handed rotation from $s$ to $s^{\prime}$ takes us along the inward normal to the antenna surface. This picture of an

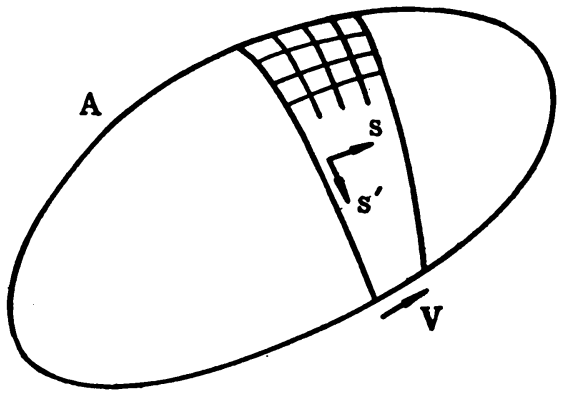

FIG. 1 antenna with a gap is illustrated on an exaggerated scale in Fig. 1.

By the "transmitting impedance" of an antenna, we shall mean, quite generally, the ratio of the current across the gap, or, more precisely, the current across one of the curves bounding the gap (and which we shall refer to simply as the "current at the gap"), to the impressed voltage (the positive sense of the current being the direction of voltage rise). This impedance will, in general, depend on what may be termed geometry of the gap (width, curvature, etc.) ${ }^{10}$ and also on the details of the applied field, i.e. different applied fields, even with the same gap and the same voltage, may give different impedances. It is reasonable to assume, however, that, within certain limits as to the size of the gap, ${ }^{11}$ the impedance is very nearly independent of the gapgeometry and of the details of the applied field, depending only on the position of the gap on the antenna surface. This is the situation which is usually tacitly assumed in engineering practice.

${ }^{7}$ It may be noticed that, in order to apply this concept in some cases, it may be necessary to extend the "antenna surface" beyond what is usually thought of as constituting the antenna. In the case of an antenna protruding through the wall of a wave-guide, for instance, the walls of the guide must (at least without further analysis) be treated as forming part of the antenna.

${ }^{8}$ J. A. Stratton and L. J. Chu, J. App. Phys., 12, 241 (1941). These authors are only concerned with a transmitting antenna, but the same procedure can obviously be adopted for a receiving antenna.

${ }^{9}$ L. Infeld, Quart. Appl. Math. (appearing shortly).

${ }^{10} \mathrm{I}$ am indebted to the referee for emphasizing to me that fact that impedance may depend on gapwidth

11 The gap must not be too small, for, as gap-width tends to zero, the investigations of Stratton and Chu (ref. 8) and Infeld (ref. 9) show that the reactive component of the current at the gap tends to infinity. On the other hand, the gap obviously must not be too large, or the details of the applied field become of importance. 
It is not the purpose of this paper to investigate the conditions under which this "practical" case may be realized. The results to be proved, however, hold whether it is realized or not (the approximations made in Secs. 4 and 5 merely require that the width of the gap be not too great in order that they may be valid).

When the antenna is receiving, we have, similarly, an "induced voltage" and a "current at the gap." When the antenna is acting as a parasite, there is, of course, no gap. Since the same antenna may be imagined to act as a transmitter, parasite, or receiver, we shall, however, speak of the "current at the gap" even in the parasite case. By this we shall mean the current at what becomes the gap when the antenna acts as transmitter or receiver. Alternatively, we may regard the parasitic antenna as a special case of the receiving antenna when the gap is "filled up" (corresponding to zero load impedance).

We shall now formulate the three mathematical problems associated with an antenna as follows:

Transmission problem. We require a solution of Maxwell's equations in the region outside the antenna surface which satisfies the following conditions:

(1) On the antenna surface the tangential components of $\mathbf{E}$ vanish except in the gap, where the following relations must be satisfied:

$$
\begin{aligned}
E_{s^{\prime}} & =0, \\
\int E_{s} d s & =-V,
\end{aligned}
$$

where the integral in (2) is taken across the gap and $V$ is the applied voltage, which is supposed given (the integral is, of course, supposed to have the same value wherever it is taken across the gap).

(2) In the surrounding medium, the tangential components of $\mathbf{E}$ and $\mathbf{H}$ are continuous across any surfaces of discontinuity of the tensors $\epsilon_{i j}, \mu_{i j}, \sigma_{i j}$.

(3) At infinity only outgoing waves are present. ${ }^{12}$

Parasite problem. We are given an incident field (whose source is assumed to be unaffected by the presence of the antenna). We require a solution of Maxwell's equations for the total field (incident plus scattered) which satisfies the following conditions:

(1) On the antenna surface, the tangential components of $\mathbf{E}$ vanish everywhere.

(2) Same as condition (2) in transmission problem.

(3) At infinity, the field must reduce to the incident field together with only outgoing (or damped) waves.

Reception problem. We are again given an incident field. We require for the total field a solution of Maxwell's equations which satisfies the following conditions:

(1) On the antenna surface, the tangential components of $\mathbf{E}$ vanish except in the gap, where the following relations must be satisfied

$$
\begin{aligned}
E_{8^{\prime}} & =0, \\
\int E_{s} d s & =Z_{L} I,
\end{aligned}
$$

12 If the medium is conducting, the corresponding condition is that the field must be space-damped at infinity. 
where $I$ is the current at the gap and $Z_{L}$ is the load impedance, i.e. the impedance at the gap looking into the connecting circuit, We regard $Z_{L}$ as given.

(2), (3) Same as (2), (3) in parasite problem.

In accordance with what has already been said, the transmission problem, as formulated above, has not, strictly speaking, a unique solution. Instead of specifying the applied voltage $V$, in condition (1), we must specify the whole applied field $E_{s}$ (a dependence of the solution on gap-geometry does not, of course, affect the uniqueness of solution). Again, in the reception problem, it is not sufficient to specify the load impedance $Z_{L}$. To make the problem determinate we must specify something as to the nature of the field $E_{s}$ within the gap. But any such possible indeterminancy in the solution of the transmission or reception problems is immaterial for our purpose.

In the case of the parasite problem, there is no gap and no question as to the uniqueness of solution arises.

3. Solution of the reception problem in terms of the solutions of the transmission and parasite problems. Thévenin's theorem. It is now easy to show that the reception problem for any antenna can be solved very simply once the transmission and parasite problems for the same antenna have been solved, it being assumed that the gap is the same for the transmission and reception problems, and that the incident field is the same for the parasite and reception problems.

Let $\left(\mathbf{E}_{1}, \mathbf{H}_{1}\right)$ be the total field (incident plus scattered) in the parasite problem, and $\left(\mathbf{E}_{2}, \mathbf{H}_{2}\right)$ the field in the transmission problem when there is unit applied voltage (or one such field in the case where the transmission problem is not uniquely determined by specifying the voltage). Then a field which is a linear combination of these two fields of the form

$$
\mathbf{E}=\mathbf{E}_{1}+\alpha \mathbf{E}_{2}, \quad \mathbf{H}=\mathbf{H}_{1}+\alpha \mathbf{H}_{2},
$$

where $\alpha$ is a properly chosen constant, gives the solution of the reception problem (or $a$ solution, if there is more than one).

For, in the first place, this field is a solution of Maxwell's equations, since all equations are supposed linear. Again, taking account of the conditions satisfied by the $\left(\mathbf{E}_{1}, \mathbf{H}_{1}\right)$ and $\left(\mathbf{E}_{2}, \mathbf{H}_{2}\right)$ fields, it is evident that conditions (2) and (3) of the reception problem, and also condition (1) except in the gap, are satisfied. Further, in the gap we have, by virtue of the conditions satisfied there for the $\left(\mathbf{E}_{1}, \mathbf{H}_{1}\right)$ and $\left(\mathbf{E}_{2}, \mathbf{H}_{2}\right)$ fields:

$$
\begin{aligned}
E_{s^{\prime}} & =0, \\
\int E_{s} d s & =\alpha \int E_{2, s} d s=-\alpha,
\end{aligned}
$$

while the current at the gap for the $(\mathbf{E}, \mathbf{H})$ field is given by

$$
I=I_{1}+\alpha I_{2}=I_{1}+\alpha / Z_{T},
$$

where $I_{1}, I_{2}$ are the currents at the gap for the $\left(\mathbf{E}_{1}, \mathbf{H}_{1}\right),\left(\mathbf{E}_{2}, \mathbf{H}_{2}\right)$ fields respectively, and $Z_{T}$ is the transmitting impedance of the antenna as defined in the previous section (in the general case, we would have to say the transmitting impedance for the particular applied field chosen). From (5), (6) and (7) we see that condition (1) of the reception problem is satisfied in the gap if $\alpha=Z_{L}\left(I_{1}+\alpha / Z_{T}\right)$, or

$$
\alpha=-Z_{L} Z_{T} I_{1} /\left(Z_{L}+Z_{T}\right) .
$$


Hence (4) gives the solution of the reception problem in terms of the solutions of the transmission and parasite problems, since (8) determines $\alpha$ in terms of known quantities, $Z_{T}$ being known from the solution of the transmission problem, $I_{1}$ (the current at the gap in the parasite problem) from the solution of the parasite problem, and $Z_{L}$ being one of the data of the reception problem. We thus see that the solution of the reception problem as formulated in the previous section is unique if, with the given gap, the transmitting impedance $Z_{T}$ is uniquely defined.

In particular, we find from (7) and (8) for the current at the gap in the reception problem

$$
I=Z_{T} I_{1} /\left(Z_{L}+Z_{T}\right),
$$

where $I_{1}$ is the current at the gap in the corresponding parasite problem. Hence the voltage which appears across the gap in the reception problem is

$$
V=-Z_{L} I=-Z_{L} Z_{T} I_{1} /\left(Z_{L}+Z_{T}\right) .
$$

The results (9) and (10) show that Thévenin's theorem, suitably interpreted, holds for an antenna. It may be remarked that these results are rigorous with the assumptions here made and apply formally to an arbitrary gap provided that the impedance $Z_{T}$ be understood in the general sense already explained.

4. Connection between the transmission and parasite problems. We have shown in the previous section that the reception problem may be solved very simply in terms of the solutions of the transmission and parasite problems. We shall now establish a connection between the transmission and parasite problems. To be precise, we shall show that if the transmission problem has been solved, then the current at the gap in the parasite problem can be found, provided: (1) the incident field can be replaced by the field of an equivalent Hertzian (infinitesimal) dipole, (2) the medium, in the neighborhood of this dipole, is homogeneous and isotropic, being characterized by a dielectric constant $\epsilon$, permeability $\mu$ and (possibly) conductivity $\sigma$. These restrictions are practically always satisfied in practice. At some distance from the source of the incident field, this field is the same as that of an appropriately chosen Hertzian dipole (or a superposition of such dipoles) and it is only the incident field in the neighborhood of the parasitic antenna which is of significance for the satisfaction of the boundary conditions in the parasite problem. Again, the medium can always, at least to a sufficient approximation, be treated as homogeneous and isotropic in the neighborhood of the equivalent Hertzian dipole.

Thus we can determine the current at the gap in the parasite problem when once the transmission problem has been solved. The results (9) and (10) then give the current at the gap and the voltage across the gap in the reception problem (with the same incident field). By varying the gap in the transmission problem we can determine the current distribution everywhere in the antenna in the parasite problem. ${ }^{13}$ Knowing this, it is usually possible to completely determine the field in the parasite problem (and hence also in the reception problem). Thus we may say that a complete solution of the transmission problem furnishes also a complete solution of the parasite and reception problems. However, in the reception problem, it is usually only the voltage induced across the gap which is of interest.

${ }^{13}$ This statement requires some modification in the case of an antenna of arbitrary shape, since what is actually furnished is the current across a closed curve on the antenna surface. For the usual case of an antenna in the shape of a wire, however, the statement remains true. 
We shall now establish the result stated above, namely, that a solution of the transmission problem furnishes also the current at the gap in the parasite problem under the conditions stated. Our method of analysis is here similar to that of Sommerfeld, ${ }^{3}$ except that we deal with a real antenna and a (fictitious) Hertzian dipole, whereas Sommerfeld considered two Hertzian dipoles. Our point of departure is the reciprocal theorem of Lorentz, ${ }^{14}$ which may be stated as follows: If $\left(\mathbf{E}_{1}, \mathbf{H}_{1}\right)$ and $\left(\mathbf{E}_{2}, \mathrm{H}_{2}\right)$ are two Maxwellian fields which have no singularities in the region external to any number of closed surfaces $U$, and which both give only outgoing (or damped) waves at infinity, then

$$
\int_{U}\left(\mathbf{E}_{1} \times \mathbf{H}_{2}\right)_{n} d S=\int_{U}\left(\mathbf{E}_{2} \times \mathbf{H}_{1}\right)_{n} d S,
$$

where $n$ denotes (say) the outward normal to the surfaces $U$. The result (11) applies if the medium outside the surfaces $U$ is of the general linear type specified at the beginning of Sec. 2. It was proved by Lorentz for the case of an isotropic medium, but the extension to the case of a crystalline medium is easily made; this is shown in the appendix.

Now let $A$ denote the antenna surface (Fig. 1) and $S$ a small sphere of radius $r$ whose center is the point $P$ where the equivalent Hertzian dipole is situated (Fig. 2).

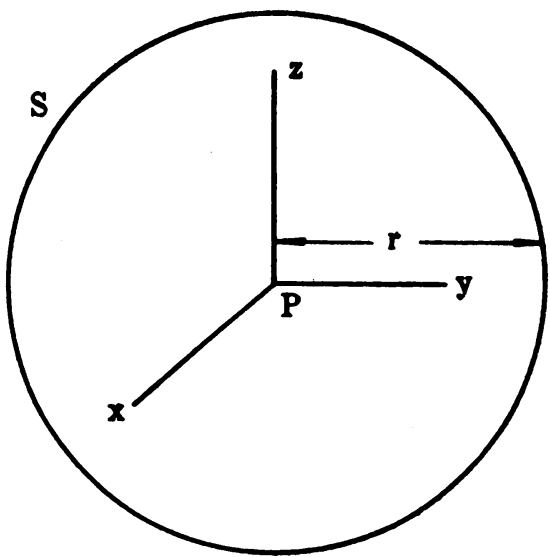

FIG. 2

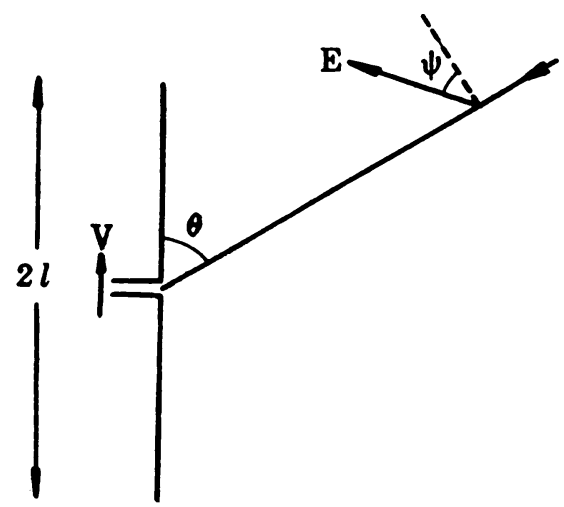

FIG. 3

Also, let $\left(\mathbf{E}_{1}, \mathbf{H}_{1}\right)$ denote the field (or $a$ field) when the antenna is transmitting with unit current at the gap and no dipole is present at $P$, and $\left(\mathbf{E}_{2}, \mathbf{H}_{2}\right)$ the field when the dipole at $P$ is transmitting and the antenna is acting as a parasite. Then, from (11), we have

$$
\int_{A+S}\left(\mathbf{E}_{1} \times \mathbf{H}_{2}\right)_{n} d S=\int_{A+S}\left(\mathbf{E}_{2} \times \mathbf{H}_{1}\right)_{n} d S,
$$

when $n$ denotes the outward normal to $A$ or $S$.

On using the boundary conditions satisfied by the $\left(\mathbf{E}_{2}, \mathbf{H}_{2}\right)$ field, we have at once

$$
\int_{A}\left(\mathbf{E}_{2} \times \mathbf{H}_{1}\right)_{n} d S=0 .
$$

${ }^{14}$ H. A. Lorentz, Amsterdamer Akad. van Wetenschappen 4, 176 (1895). 
To calculate the other integral over $A$ in (12), we introduce orthogonal curvilinear coordinates $(\xi, \eta)$ in the gap, the curves $\eta=$ constant being in the " $s$ "-direction, and the curves $\xi=$ constant in the " $s$ " direction (Fig. 1). Let the line element in the gap be

$$
d s^{2}=h_{1}(\xi, \eta) d \xi^{2}+h_{2}(\xi, \eta) d \eta^{2} .
$$

Then, taking account of the boundary conditions satisfied by the $\left(\mathbf{E}_{1}, \mathbf{H}_{1}\right)$ field, we have

$$
\int_{A}\left(\mathbf{E}_{1} \times \mathbf{H}_{2}\right)_{n} d S=\iint E_{1, \xi} H_{2, \eta} h_{1} h_{2} d \xi d \eta,
$$

where the double integral is taken over the surface of the gap, and the limits for $\xi, \eta$ are constants.

We shall now assume that we can neglect the dependence of $H_{2, \eta}$ and $h_{2}$, or at least of the product $H_{2, \eta} h_{2}$, on $\xi$. This amounts to neglecting the variation of the current density across the gap when the antenna is acting as parasite, and to neglecting the width of the gap in comparison with the radius of curvature of the $s$-curves. The former involves an error which would usually be of the order $w / \lambda$, where $w$ is the width of the gap and $\lambda$ the wave-length. The latter involves an error of the order $w / R$ where $R$ is the radius of curvature of the $s$-curves.

With this assumption we now have

$$
\begin{aligned}
\int_{A}\left(\mathbf{E}_{1} \times \mathbf{H}_{2}\right)_{n} d S & =\int E_{1, \xi} h_{1} d \xi \cdot \int H_{2, \eta} h_{2} d \eta \\
& =\int E_{1,8} d s \cdot \oint H_{2,8^{\prime}} d s^{\prime},
\end{aligned}
$$

where the first integral in the last expression in (13a) is taken across the gap and the second integral is taken round the gap along any one of the $s^{\prime}$-curves. The first integral is equal to the voltage across the gap when the antenna is transmitting, while the second integral (since the antenna is perfectly conducting) is equal to $4 \pi / c$ times the current at the gap when the antenna is acting as a parasite (the difference between the currents across any one of the $s^{\prime}$-curves is neglected, according to our assumption). Remembering that in the $\left(\mathbf{E}_{1}, \mathbf{H}_{1}\right)$ field, the applied voltage is assumed to give unit current at the gap, we thus finally have

$$
\int_{A}\left(\mathbf{E}_{1} \times \mathbf{H}_{2}\right)_{n} d S=\frac{4 \pi}{c} Z_{T} I_{1},
$$

where $Z_{T}$ is the transmitting impedance of the antenna, and $I_{1}$ is the current at the gap in the $\left(\mathbf{E}_{2}, \mathbf{H}_{2}\right)$ field.

We shall now calculate the integrals over $S$ in (12) when we proceed to the limit $r \rightarrow 0$. For this purpose, let us take axes (Fig. 2) with the origin at $P$, and the $z$-axis in the direction of the axis of the dipole. Then, since the medium is supposed to be homogeneous and isotropic in the neighborhood of $P$, we have for the $\left(\mathbf{E}_{2}, \mathbf{H}_{2}\right)$ field on the sphere $S$, correct to terms of order $1 / r^{2}$ :

$$
\left.\begin{array}{lll}
E_{2, x}=3 M z x / r^{5}, & E_{2, y}=3 M z y / r^{5}, & E_{2, z}=M\left(\frac{3 z^{2}}{r^{5}}-\frac{1}{r^{3}}\right), \\
H_{2, x}=i k \epsilon^{\prime} M y / r^{3}, & H_{2, y}=-i k \epsilon^{\prime} M x / r^{3}, & H_{2, z}=0,
\end{array}\right\}
$$


where

$$
k=\omega / c, \quad \epsilon^{\prime}=\epsilon+4 \pi i \sigma / \omega,
$$

and $M$ denotes the strength ${ }^{15}$ of the dipole. We thus see that $\mathbf{E}_{2}$ is of order $1 / r^{3}$, whereas $\mathrm{H}_{2}$ is of order $1 / r^{2}$, on $S$. Hence, to calculate the limit of the integrals over $S$ occurring in (12), it is sufficient to put

$$
\mathrm{E}_{1}=\left(\mathrm{E}_{1}\right)_{P},
$$

but it is not sufficient to put $\mathbf{H}_{1}=\left(\mathbf{H}_{1}\right)_{P}$. We must, instead, put

$$
H_{1, x}=\left(H_{1, x}\right)_{P}+x\left(\frac{\partial H_{1, x}}{\partial x}\right)_{P}+y\left(\frac{\partial H_{1, x}}{\partial y^{\prime}}\right)_{P}+z\left(\frac{\partial H_{1, x}}{\partial z}\right)_{P},
$$

with similar expressions for $H_{1, y}, H_{1, z}$.

Using (15), (17), (18), the calculation of the integrals over $S$ is straightforward and similar to the corresponding calculations of Sommerfeld, ${ }^{3}$ except that he omitted to take account of the variation of $\mathbf{H}_{1}$ over $S$ (in our notation), as expressed by (18). ${ }^{16}$ We thus find that, as $r \rightarrow 0$,

$$
\begin{aligned}
& \int_{S}\left(\mathbf{E}_{1} \times \mathbf{H}_{2}\right)_{n} d S=\frac{8}{3} \pi i k \epsilon^{\prime} M\left(E_{1, z}\right)_{P}, \\
& \int_{S}\left(\mathbf{E}_{2} \times \mathbf{H}_{1}\right)_{n} d S=\frac{4}{3} \pi M\left(\frac{\partial H_{1, y}}{\partial x}-\frac{\partial H_{1, x}}{\partial y}\right)_{P}=-\frac{4}{3} \pi i k \epsilon^{\prime} M\left(E_{1, z}\right)_{P .}
\end{aligned}
$$

The last step in (20) follows from the Maxwell equation

$$
\operatorname{curl} \mathbf{H}_{1}=-i k \epsilon^{\prime} \mathbf{E}_{1},
$$

which, by supposition, holds in the neighborhood of $P$.

Substituting (13), (14), (19), (20) in (12), we now have

$$
Z_{T} I_{1}=-i \omega \epsilon^{\prime} M E_{d},
$$

where $E_{d}=\left(E_{1, z}\right)_{P}$ denoted the component along the axis of the equivalent dipole of the electric field generated when the antenna is transmitting with unit current at the gap. We repeat here that $M$ is the strength of the equivalent Hertzian dipole, and $\epsilon^{\prime}$ the "complex dielectric constant," defined by (16), of the medium at the position of the equivalent dipole. This is the required result. For, if the transmission problem has been solved, $Z_{T}$ and $E_{d}$ are known, and (21) then gives $I_{1}$, the current at the gap in the parasite problem. In general, both $Z_{T}$ and $E_{d}$ might depend on the details of the applied field, but (21) shows that, to the approximation with which (13a) holds, the ratio $Z_{T} / E_{d}$ is independent of such details. ${ }^{17}$

The position and strength of the equivalent dipole can usually be assigned very

16 The "strength" of the dipole can be defined by saying that the field is given by (15) in the immediate neighborhood of the dipole; alternatively, by saying that the field is that derived from a Hertz vector whose components are $\left(0,0, M \exp \left(i k \sqrt{\epsilon^{\prime} \mu} r\right) / r\right.$.

${ }^{16}$ Sommerfeld thus obtained the incorrect result zero for the integral in (20). This error does not appear in his final result, since he was dealing with two Hertzian dipoles. It is essential to correct it in the present calculations, however.

17 The case of unit current at the gap has been chosen simply for convenience. In general, the ratio $V / E_{d}^{\prime}$ appears in place of the ratio $Z_{T} / E_{d}$ in (21), where $V$ is the applied voltage and $E_{d}^{\prime}$ the corresponding value of $E_{d}$. 
easily when once the incident field is given. The assignment is usually by no means unique. In the common case of a plane-polarized wave, the equivalent dipole can conveniently be taken at a large distance from the antenna, and the strength of the dipole expressed in terms of the amplitude of the incident wave. If the incident field is the same as that of two equivalent Hertzian dipoles which are out of phase, as is the case with an elliptically-polarized wave, we need merely superpose the fields of each dipole separately, obtaining in place of (21)

$$
Z_{T} I_{1}=-i \omega \epsilon^{\prime}\left(M_{1} E_{d 1}+M_{2} E_{d 2}\right),
$$

where the suffixes 1 and 2 on the right-hand side refer to the two equivalent dipoles.

Let us, for instance, apply (21) to the case where the whole medium is homogeneous and isotropic, being characterized by constants $\epsilon, \mu, \sigma$, and where the incident field is that of a plane-polarized wave. Let us take polar coordinates $(R, \theta, \phi)$ with the origin in the neighborhood of the antenna, and let us take the equivalent dipole at a large distance from the antenna in the direction from which the incident wave arrives, the axis of the dipole being in the direction of the electric vector of the incident wave. Then if $E_{\text {ine }}$ is the (complex) amplitude of the electric vector in the incident wave at the origin, we have

$$
E_{\mathrm{inc}}={k^{\prime}}^{2} M e^{i k^{\prime} R} / R,
$$

where $\epsilon^{\prime}$ is again the "complex dielectric constants" defined by (16), and $k^{\prime}=\sqrt{\epsilon^{\prime} \mu} k$. Again, for any shape of antenna, we can always express $E_{d}$ in the form

$$
E_{d}=i \mu f(\theta, \phi, \psi) e^{i k^{\prime} R} / c R,
$$

where $\psi$ is an azimuthal angle with respect to the radius vector which defines the direction of the electric vector in the incident wave, and $f(\theta, \phi, \psi)$ is a dimensionless function which can be calculated when the transmission problem of the antenna has been solved. This function determines the directional and polarization characteristics of the antenna when transmitting. Substituting for $M$ and $E_{d}$ from (23), (24) in (21), we have for the current at the gap in the parasite problem in the present case

$$
I_{1}=E_{\text {inc }} f(\theta, \phi, \psi) / k Z_{T} .
$$

Equation (25) gives the current at the gap in the parasite problem in terms of the amplitude of the incident wave and quantities which are known from the solution of the transmission problem. From (10), we then have for the voltage induced at the gap in the reception problem

$$
V=-Z_{L} E_{\text {inc }} f(\theta, \phi, \psi) / k\left(Z_{L}+Z_{T}\right) .
$$

Consider, for example, the well-known case of a linear antenna of length $2 l$, with the gap at the middle point. Let us take $\psi$ to be the angle between the electric vector of the incident wave and the plane containing the antenna and the direction of the incident wave (Fig. 3). Also let us take the origin at the center of the antenna with the polar axis along the antenna (in the direction in which the voltage rise is measured), so that $\theta$ is the angle which the direction of the incident wave makes with the antenna. Then, with the usual simplified treatment and the assumption of a sinusoidal current in the antenna for the transmission problem, we have for the function $f$ in (24) 


$$
f(\theta, \phi, \psi)=2 \frac{\cos (k l \cos \theta)-\cos k l}{\sin k l \sin \theta} \cos \psi
$$

Hence (26) now gives for the voltage induced at the gap in the reception problem

$$
V=-\frac{2}{k} \frac{Z_{L} E_{\text {ino }}}{Z_{L}+Z_{T}} \frac{\cos (k l \cos \theta)-\cos k l}{\sin k l \sin \theta} \cos \psi .
$$

The result (28) is in agreement with one obtained by Hallén ${ }^{18}$ by a direct treatment of the reception problem, to the order to which (27) is true, i.e., with the usual assumption of a sinusoidal current in the antenna when transmitting. A more accurate treatment of the transmission problem would yield a more accurate expression than (27) for the function $f$, and hence also a more accurate expression than (28) for the induced voltage in the reception problem.

5. Reciprocal relations between two antennas. The results of the two preceding sections establish relations between the performance of a single antenna when acting as transmitter, parasite or receiver. They deal with the fields generated or received by the antenna, and make no mention of the second antenna which is required to detect the transmitted field, or to generate the incident field. Such relations would seem to be those of most use in establishing a connection between the transmitting and receiving properties of antennas. The more usual results establishing this connection, however, such as have been given by Carson ${ }^{1}$ and Sommerfeld, ${ }^{3}$ relate to the performance of two antennas. We shall now establish such "reciprocal relations."

Consider two antennas, $A_{1}, A_{2}$, with gaps $G_{1}, G_{2}$ (Fig. 4). Let us apply the Lorentz
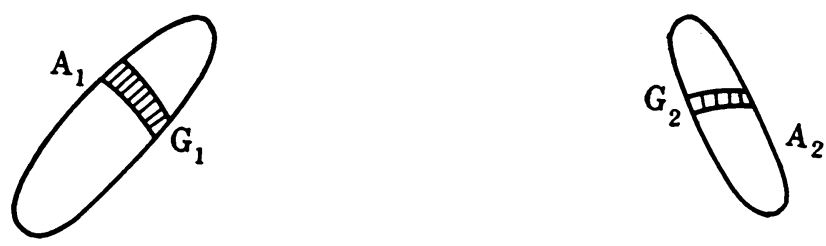

FIG. 4

relation (11) to the region outside $A_{1}, A_{2}$, taking for $\left(\mathrm{E}_{1}, \mathrm{H}_{1}\right)$ the field (or $a$ field) when $A_{1}$ is transmitting with an applied voltage $V_{1}$ and $A_{2}$ acting as parasite, and for $\left(\mathrm{E}_{2}, \mathrm{H}_{2}\right)$ the field when $A_{2}$ is transmitting with an applied voltage $V_{2}$ and $A_{1}$ acting as parasite. By an exactly similar method to that used in deriving (13a) and (14), and with a similar approximation (i.e. neglecting terms of order $w / \lambda$ and $w / R$ ) we now have:

$$
\begin{aligned}
& \int_{A_{1}}\left(\mathbf{E}_{1} \times \mathrm{H}_{2}\right)_{n} d S=\frac{4 \pi}{c} I_{1} V_{1} \\
& \int_{A_{2}}\left(\mathbf{E}_{2} \times \mathrm{H}_{1}\right)_{n} d S=\frac{4 \pi}{c} I_{2} V_{2}, \\
& \int_{A_{2}}\left(\mathbf{E}_{1} \times \mathrm{H}_{2}\right)_{n} d S=\int_{A_{1}}\left(\mathbf{E}_{2} \times \mathrm{H}_{1}\right)_{n} d S=0
\end{aligned}
$$

${ }^{18}$ E. Hallén, Nova Acta Reg. Soc. Upsaliensis 11, No. 5 (1938). 
where $I_{1}^{\prime}$ is the current induced at $G_{1}$ in the $\left(\mathbf{E}_{2}, \mathbf{H}_{2}\right)$ field, and $I_{2}$ the current induced at $G_{2}$ in the $\left(\mathbf{E}_{1}, \mathbf{H}_{1}\right)$ field. Substituting (29), (30), (31), in (11) we obtain ${ }^{19}$

$$
V_{1} I_{1}=V_{2} I_{2} \text {. }
$$

This is the required reciprocal relation. We may state the content of the relation in words as follows: Let $A_{1}, A_{2}$ be two antennas with gaps $G_{1}, G_{2}$ respectively. Then the current induced at $G_{1}$ when $A_{2}$ is transmitting and $A_{1}$ acting as parasite is equal to the current induced at $G_{2}$ when $A_{1}$ is transmitting and $A_{2}$ acting as parasite, providing that the applied voltages in the two cases are equal. ${ }^{20}$ This is the more precise form of Carson's ${ }^{1}$ original reciprocal theorem.

More generally, if each antenna acts as a receiver instead of a parasite when the other is transmitting, let $Z_{T 1}$ be the transmitting impedance of $A_{1}$, and $Z_{L 1}$ the load impedance when $A_{1}$ is receiving, the gap being at $G_{1}$ in both cases. Let $Z_{T 2}, Z_{L 2}$ denote similar quantities for $A_{2}$, the gap being at $G_{2}$. Then (9) and (32) lead to the relation

$$
\left(1+Z_{L 1} / Z_{T 1}\right) V_{1} I_{1}=\left(1+Z_{L 2} / Z_{T 2}\right) V_{2} I_{2}
$$

in place of (32), where $I_{1}$ is now the current induced at $G_{1}$ when $A_{2}$ is transmitting with applied voltage $V_{2}$ and $A_{1}$ receiving, and $I_{2}$ is the current induced at $G_{2}$ when $A_{1}$ is transmitting with applied voltage $V_{1}$ and $A_{2}$ is receiving. If, instead of induced currents, we deal with induced voltages, the relation (33) becomes

$$
\left(Z_{T_{1}}+Z_{L 1}\right) V_{1} v_{1}=\left(Z_{T 2}+Z_{L 2}\right) V_{2} v_{2}
$$

where $v_{1}$ is the voltage induced at $G_{1}$ when $A_{1}$ is receiving and $A_{2}$ transmitting, and $v_{2}$ is the voltage induced at $G_{2}$ when the roles of transmitter and receiver are interrhanged. $V_{1}, V_{2}$ denote, as before, the applied voltages.

We may here briefly refer to an apparent contradiction of the relation (32) which has been put forward by Ballantine. ${ }^{2}$ It was originally given by this author in criticism of Sommerfeld's ${ }^{3}$ form of reciprocal theorem; but it would apply equally well to the more exact form of the theorem given above. Let the antenna $A_{1}$ considered in the relation (32) be a linear whole-wave antenna which, when transmitting, has a sinusoidal current distribution which is antisymmetrical about the middle point ("complete positive and negative current loops"), as shown in Fig. 5. Let the second antenna $A_{2}$, which may be of arbitrary shape, ${ }^{21}$ be situated at a large distance from $A_{1}$ and in a direction perpendicular to it (Fig. 5). Then, when $A_{1}$ is transmitting, with the assumed current distribution, the field-strength generated is zero in the direction of $A_{2},{ }^{22}$ so that no current will be induced anywhere in $A_{2}$ when $A_{2}$ is acting as parasite. On the other hand when $A_{2}$ is transmitting and $A_{1}$ acting as parasite it is certainly

19 In the notation more generally used, $I_{1}$ and $I_{2}$ are interchanged in (32).

${ }^{20}$ It should be recalled that we are dealing with complex amplitudes. What is implied, therefore, is that if the (real) amplitudes of the applied voltages are equal in the two cases, then both the current amplitudes and the phase differences between induced current and applied voltage are also equal. The frequencies must, of course, be the same in the two cases.

${ }^{21}$ Ballantine supposes that the second antenna is exactly similar to the first (but not parallel to it) in order to comply with the conditions of Sommerfeld's theorem. But this restriction is of no importance in the present connection.

22 The longitudinal component of field-strength is actually only zero when terms of order higher than $1 / r$, where $r$ denotes distance from the antenna, are neglected. This is all that is required for the argument, however. 
not true in general that no current is induced in $A_{1}$. This contradicts the reciprocal relation (32).

The explanation is that a completely antisymmetrical current distribution of the type postulated when $A_{1}$ is transmitting cannot occur. Ballantine makes no mention as to where the antenna is supposed to be fed. If fed at the center, the current distribution is symmetrical, not antisymmetrical. If fed at some other point, well away from the center, the current distribution will be approximately of the type postulated, but not exactly, since the usual sinusoidal theory is not exact. The field-

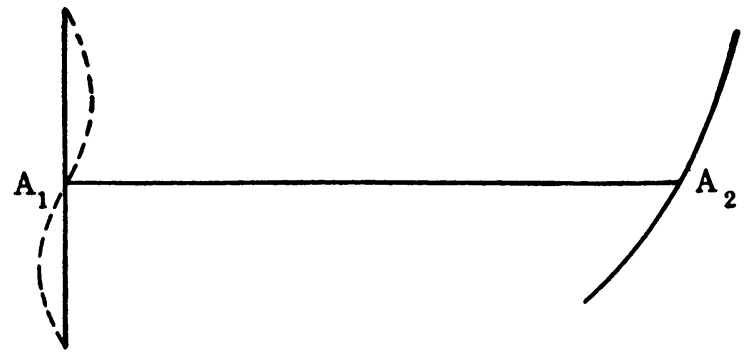

FIG. 5

strength generated by $A_{1}$ in the direction of $A_{2}$ is thus not zero, but is comparatively small. The reciprocal relation then tells us (quite correctly) that $A_{1}$ will be comparatively weakly affected when $A_{2}$ is transmitting.

6. Are the directional properties (polar diagrams) of an antenna identical for transmission and reception? It is commonly stated that the directional properties of an antenna, as exhibited in a polar diagram, are identical for transmission and reception. It is of interest to see whether the results of the two preceding sections confirm this or not.

The reciprocal relation (32) shows at once that, in a certain sense, the directional properties are identical. Consider an antenna $A$ and a large number of other antennas (which need not necessarily be identical) situated at a large distance from $A$ in different directions. Then we might define the polar diagram of $A$ for transmission by means of the currents induced at certain "gaps" in these antennas when they act as parasites, and the polar diagram of $A$ for reception by means of the currents induced at the gap in $A$ when $A$ acts as parasite and any one of the distant antennas is transmitting, the same voltage being applied in every case. The relation (32) then shows that the polar diagrams are the same.

Again, instead of considering the antennas as acting as parasites, we might consider them as acting as receivers, the load impedance being the same for all the distant antennas, and use the induced voltages, rather than the parasitic currents, to define the polar diagrams for transmission and reception. The relation (34) then shows that the polar diagrams are identical $\left(v_{1} / v_{2}\right.$ constant for $V_{1} / V_{2}$ constant) only if the transmission impedances $Z_{T}$ are identical for all the distant antennas. If all these antennas are identical, this would only be the case if the whole medium is homogeneous and isotropic; otherwise, the equality of the transmission impedances could be achieved by proper choice of the distant antennas.

Neither of the above ways of defining the polar diagrams is very satisfactory, however, and neither of them corresponds to what is usually adopted in practice. The polar diagram for transmission is usually defined by means of the field generated, or 
the intensity, at distant points, and the polar diagram for reception by means of the voltage induced by, or the power absorbed from, an incident wave of given strength arriving from different directions.

For definiteness we shall, in an endeavor to treat the matter quite generally, define the polar diagrams of an antenna for transmission and reception (with the same gap) as follows: draw a large sphere $S^{23}$ surrounding the antenna $A$. Then the polar diagram for transmission is constructed by making the length of the radius vector in any direction in the diagram proportional to the maximum amplitude of the electric field-strength generated (for constant applied voltage ${ }^{23 a}$ ) at the point on $S$ which lies in this direction. For reception, we suppose that the source of the incident field can be replaced by an equivalent Hertzian dipole (cf. Sec. 4) at some (variable) point $P$ on $S$, the axis of the dipole being in the direction in which the electric field-strength generated at $P$ when $A$ is transmitting is a maximum. ${ }^{24}$ The strength of the equivalent dipole is then adjusted so that the maximum amplitude of the electric field-strength in the incident field at $A$ is the same for all points $P$ on $S$. Then the polar diagram for reception is constructed by making the length of the radius vector in the direction of $P$ in the diagram proportional to the voltage induced in $A$ when the equivalent dipole is at $P$, the gap in $A$ being the same as for transmission and the load impedance being kept constant. It is perhaps worth pointing out that the direction of $P$ (i.e., of the source) is not necessarily the same as the direction of arrival of the incident wave if inhomogeneities of the medium are present. The incident field at the antenna need not, in fact, be a plane wave at all, in which case we cannot even speak of the direction of the incident wave.

With these definitions, it follows from (21) and (10) that the polar diagrams for transmission and reception are identical if, and only if, the expression $\left|\epsilon^{\prime} M / E_{\text {inc }}\right|$ is constant for all directions from the antenna (i.e. for all points on the distant sphere), where $\left|E_{\text {ino }}\right|$ denotes the maximum amplitude of the electric field-strength in the incident field when the strength of the equivalent Hertzian dipole is $M$, and $\epsilon^{\prime}$ is the complex dielectric constant of the medium at the position of this dipole.

The expression $\left|\epsilon^{\prime} M / E_{\text {ino }}\right|$ is certainly constant if the whole medium is homogeneous and isotropic, for $\epsilon^{\prime}$ and $M / E_{\text {inc }}$ are then both constant. It is also constant, for instance, if we are considering transmission and reception over a perfectly conducting earth, or over an imperfectly conducting, but homogeneous, earth. It is not in general constant, however, when inhomogeneities are present-e.g. for a heterogeneous imperfectly conducting earth.

We conclude, therefore-at least with the above definitions of the polar diagrams-that the identity of the polar diagrams is not a general property of antennas when inhomogeneities of the medium are present, although the diagrams are probably identical, or approximately so, in practically-occurring cases.

We may note incidentally that (21) and (10) show that the voltage induced in an antenna by an equivalent Hertzian dipole of given moment, and situated at a given point, is a maximum when the axis of the dipole lies in the direction in which the electric field-strength generated at that point when the antenna is transmitting is a

\footnotetext{
${ }^{23}$ In general, the polar diagrams obtained from our definitions may depend on the radius of this sphere. In practice they will usually not do so, however, provided that the sphere is large.

23a The diagram thus obtained is independent of the applied voltage by (21) and Footnote 17.

${ }^{24}$ This somewhat arbitrary choice is made so as to render conditions most favorable for the identity of the two polar diagrams. It implies, of course, a certain restriction on the source of the incident wave.
} 
maximum. When the whole medium is homogeneous and isotropic, this means that the voltage induced by a plane-polarized incident wave is a maximum, other things being equal, when the polarization of the incident wave is the same as that of the wave generated when the antenna is transmitting (or, in the general case, where the generated wave is elliptically-polarized, when the electric vector of the incident wave is in the direction of the major axis of the ellipse described by the electric vector of the generated wave).

7. Extension of the results to include imperfect conductivity of the antenna. So far, we have dealt entirely with the case where the antenna is perfectly conducting. We shall now indicate briefly how the results can be extended to include the case of an imperfectly conducting antenna.

We shall now adopt the following "mathematical model" for a transmitting or receiving antenna (cf. Sec. 2): there is a "gap" as before, and the conditions (1), (2) or (3a), (3b) again hold; but we now postulate in addition that over the ends of the gap (i.e., the cross-sections of the antenna through the curves marking the position of the gap) the tangential components of $\mathbf{E}$ vanish, both in transmission and reception. The "gap" is thus now a kind of "box," and the surrounding medium now includes the interior of the antenna which lies outside this "box." The transmission and reception problems for an antenna now require a solution of Maxwell's equations in the region outside the "box," subject to the proper boundary conditions in this region, which are the same as before, and subject to the appropriate surface boundary conditions on the box. The latter are the same as before, with the additional condition that the tangential components of $\mathbf{E}$ vanish over the ends of the box. The condition that the tangential components of $\mathbf{E}$ vanish on the surface of the antenna now disappears. In the parasite problem, there is no "box" and hence, of course, no conditions to be applied to its surface.

We shall now make the followed additional assumptions:

(1) The displacement current at the gap is negligible compared with the conduction current.

(2) When the antenna is acting as a parasite, the integral $\int E_{8} d s$, taken across the gap (Fig. 1), is negligible.

(3) When the antenna is acting as a parasite, the component $E_{s^{\prime}}$ (Fig. 1) and the tangential components of $\mathbf{E}$ over the ends of the gap are negligible.

The only one of these assumptions to which exception might be taken is the last, which is certainly not true for an antenna of arbitrary shape and with an arbitrary gap. It is, however, very approximately true for the usual wire-shaped antenna, so that it seems reasonable to adopt it as a general assumption, particularly in view of the somewhat arbitrary character of our "box model."

It is now readily verified that the general results of sections $3-5$ can be proved as before, by merely substituting the surface of the box for the surface of the antenna (not all the assumptions (1)-(3) above are needed in the proof of each result). The discussion of section 6 then applies as before. The formula (27) (and hence (28)), which relates to a special problem, requires modification, of course, if the imperfect conductivity of the antenna is taken into account.

\section{APPENDIX}

We shall here establish the Lorentz relation (11) when the medium outside the surfaces $U$ is anisotropic, being characterized by the symmetric tensors $\epsilon_{i j}, \mu_{i j}, \sigma_{i j}$, 
which may be arbitrary functions of position (cf. Sec. 2). Adopting for a moment the indicial notation, so that, e.g., we write $\left(E_{1}, E_{2}, E_{3}\right)$ in place of $\left(E_{x}, E_{y}, E_{z}\right)$, Maxwell's equations for the steady state (with the time-factor $e^{-i \omega t}$ ), and for the postulated type of medium, can be written

$$
\begin{aligned}
& (\operatorname{curl} \mathbf{E})_{i}=-\frac{i \omega}{c} \sum_{j} \mu_{i j} H_{j}, \\
& (\operatorname{curl} \mathbf{H})_{i}=\frac{i \omega}{c} \sum_{j} \epsilon_{i j} E_{i}+\frac{4 \pi}{c} \sum_{j} \sigma_{i j} E_{i},
\end{aligned}
$$

for $i=1,2,3$, the summations running in all cases from 1 to 3 .

Suppose now we have two frelds $\left(\mathbf{E}^{(1)}, \mathbf{H}^{(1)}\right)$ and $\left(\mathbf{E}^{(2)}, \mathbf{H}^{(2)}\right)$ which satisfy these equations. Then we can write:

$$
\begin{aligned}
\left(\operatorname{curl} \mathbf{E}^{(1)}\right)_{i} & =\sum_{j} \alpha_{i j} H_{j}^{(1)}, \\
\left(\operatorname{curl} \mathbf{H}^{(1)}\right)_{i} & =\sum_{i} \beta_{i j} E_{j}^{(1)}, \\
\left(\operatorname{curl} \mathbf{E}^{(2)}\right)_{i} & =\sum_{i} \alpha_{i j} H_{i}^{(2)}, \\
\left(\operatorname{curl} \mathbf{H}^{(2)}\right)_{i} & =\sum_{i} \beta_{i j} E_{j}^{(2)},
\end{aligned}
$$

where

$$
\alpha_{i j}=-\frac{i \omega}{c} \mu_{i j}, \quad \beta_{i j}=\frac{i \omega}{c} \epsilon_{i j}+\frac{4 \pi}{c} \sigma_{i j} .
$$

Multiplying (A1), (A2), (A3, (A4) by $H_{i}^{(2)}, E_{i}^{(2)},-H_{i}^{(1)},-E_{i}^{(1)}$ respectively, adding, and summing with respect to $i$, we obtain, on using the identity $\mathbf{A} \cdot \operatorname{curl} \mathbf{B}-\mathbf{B} \cdot$ curl $A=\operatorname{div}(B \times A)$ :

$$
\begin{aligned}
\operatorname{div}\left[\mathbf{E}^{(1)} \times \mathbf{H}^{(2)}-\mathbf{E}^{(2)}\right. & \left.\times \mathbf{H}^{(1)}\right] \\
& =\sum_{i, j}\left[\alpha_{i j}\left(H_{j}^{(1)} H_{i}^{(2)}-H_{j}^{(2)} H_{i}^{(1)}\right)+\beta_{i j}\left(E_{j}^{(1)} E_{i}^{(2)}-E_{j}^{(2)} E_{i}^{(1)}\right)\right] \\
& =\sum_{i, j}\left[\left(\alpha_{i j}-\alpha_{j i}\right) H_{j}^{(1)} H_{i}^{(2)}+\left(\beta_{i j}-\beta_{j i}\right) E_{j}^{(1)} E_{i}^{(2)}\right]=0,
\end{aligned}
$$

since, from (A5), the tensors $\alpha_{i j}, \beta_{i j}$ are symmetric.

Hence, using the notation of (11),

$$
\operatorname{div}\left(\mathbf{E}_{1} \times \mathrm{H}_{.2}\right)=\operatorname{div}\left(\mathrm{E}_{2} \times \mathrm{H}_{1}\right) .
$$

From (A6) the required relation (11) follows in the usual way by integrating each side of the equation over the region bounded by the surfaces $U$ and an infinite sphere, and applying Green's theorem. This sphere, and also any surfaces of discontinuity, give no contributions to the surface integrals on account of the boundary conditions (outgoing waves at infinity; continuity of tangential components of $\mathbf{E}$ and $\mathbf{H}$ at a surface of discontinuity). 